\title{
Taking Off the Mask: Impostorism and Medical Education
}

Mark B. Stephens, MD, MS

PRiMER. 2022;6:5.

Published: 2/23/2022 | DOI: 10.22454/PRiMER.2022.527933

\section{Abstract}

Introduction: Professional identity formation is the complex and iterative process by which medical students learn to think, act, and feel like physicians.

Methods: Using mask making, we iteratively explored changes in student perceptions of their identity across time during medical school.

Results: Themes of impostorism, uncertainty, and identity progression/integration were identified.

Conclusion: Mask making represents a unique method to examine fundamental themes in identity formation for medical students.

\section{Introduction}

In recent years, the use of art and art making has increasingly been incorporated as part of formal medical school curricula. ${ }^{1}$ Inclusion of arts-based activities has been shown to increase empathy, promote tolerance of ambiguity, and promote teamwork in medical students. ${ }^{2}$ At the Penn State College of Medicine, University Park Regional Campus (UPRC), mask making has been used to examine identity in medical students and teams of medical professionals. ${ }^{3-5}$ The process of mask making offers individuals a safe space to explore, analyze, and synergize developing identities in a visual context. ${ }^{3}$ Through this visually creative modality, individuals are given the opportunity to explore and express their identity as a developing professional. ${ }^{6}$

One framework that has been used to describe identity formation in multiple professional sectors is Kegan's Adult Developmental Theory. ${ }^{7}$ This model suggests that individuals continuously seek to find meaning in the context of self and others through sequential (and somewhat predictable) stages. Cruess and colleagues extended this model to medical education, adding the importance of socialization as students move from being peripheral participants to full members of the community of practice of medicine. ${ }^{8}$ When supported by mentors and appropriate institutional norms and cultures, the process of professional identity formation (PIF) generally leads to a holistic sense of self that includes personal and professional elements of identity. If there are perturbations to the process (eg, elements of the hidden curriculum), the result is identity dissonance and a delayed feeling of an authentic professional self. ${ }^{9}$

As students navigate their personal PIF journey, the impostor phenomenon (also referred to as imposterism or the impostor syndrome) is an increasingly well-recognized situation wherein highly successful individuals 
attribute their success to external factors (such as luck, knowing the right people, or being in the right place at the right time. ${ }^{10}$ Several recent reviews ${ }^{11,12}$ found the impostor phenomenon to be present in upwards of $80 \%$ of health care professionals. The present study highlights thoughts and emotions related to impostorism expressed iteratively through the creation (and reflection upon) these masks.

\section{Methods}

Following Institutional Review Board approval, all 11 students graduating from UPRC in the class of 2021 were invited to participate. Each year, as part of the curriculum at UPRC, students in this class were asked to create a mask exploring their sense of identity as a medical professional. Students created their initial mask during orientation to their first year of medical school. They then completed a mask at the end of each academic year. Just prior to graduation, students were asked to create their final mask (for a total of five masks). Using a series of guided prompts (Table 1), students were then asked to compare and contrast their final mask with their previous masks. Students were specifically challenged to think about changes in their sense of professional identity during their time in medical school. To engage in intentional thematic analysis, freeresponse answers to these prompts were then qualitatively analyzed using inductive coding, iteration and constant comparison. ${ }^{13}$

\section{Results}

Five of 11 graduating students consented to participate. Overall, three primary themes emerged from participant responses (Table 2). The most prominent and consistent theme centered around the impostor phenomenon. Impostorism is common in medical education, ${ }^{14}$ and the mask-making exercise afforded students the explicit opportunity to identify and directly address these feelings. Respondents explicitly noted the pervasiveness of impostorism. Overall feelings of impostorism were so strong in one participant that they contemplated withdrawing from medical school. The second and third themes were consistent with prior iterative reflections we reported on developing medical student identity across time. ${ }^{6}$ One of these themes was uncertainty. Participants particularly emphasized uncertainty in terms of their evolving roles as medical professionals. They also described uncertainty with the mask making process itself, particularly a common thread of lacking artistic confidence and worrying about what others might think about their mask (and potentially their identity as a medical professional). The final theme focused on identity progression and integration. Participants richly described their developing sense of identity at both the individual (micro) level and the systems (macro) level. As medical school progressed, participants felt more comfortable in their role as a medical professional. They also recognized that the process likely would start over with their new roles as residents. A representative mask is shown in Figure 1.

\section{Discussion}

Mask making is a useful tool to explore identity formation in medical education. ${ }^{5}$ As such, the theme or message of the mask is dictated by the context of the mask-maker's life experiences and related to salient issues at hand. Mask making offers individuals a safe space to explore, analyze, and synergize developing identities. This small study builds on our previous work highlighting the iterative nature of professional identity formation. ${ }^{6}$ It adds to existing literature ${ }^{15}$ highlighting impostorism as a central theme that students deal with during medical school as they learn to think, act, and feel like physicians. Furthermore, while small in scope, the results of this study suggest that while the impostor phenomenon is common in medical education, students are able to recognize and articulate their feelings of impostorism and work through those feelings over time.

While limited by small sample size and being a single-institution study, this longitudinal pilot study 
demonstrates the potential use of art and mask making to provide powerful insight into the complex phenomenon of impostorism in medical education. Key findings include the pervasiveness of impostorism, the ubiquity of uncertainty, and the iterative process of integrating multiple elements into an overall sense of self. Knowing the wide prevalence of the impostor phenomenon, we plan to use the findings of this study to prospectively examine the use of mask making as a strategy to mitigate impostorism in medicine and promote healthy longitudinal professional identity formation.

\section{Tables and Figures}

\section{Table 1: Reflective Prompts}

1. How do you feel your professional self-identity has changed during your time in medical school?

2. Looking back on your masks, what features of your professional identity do each express?

3. How has the mask-making process shaped your view of professional identity formation?

4. What common features do you notice in your masks?

5. If given the chance, what would your fourth-year mask say to your first-year mask in the context of developing a holistic professional identity?

6. What other observations do you have about your masks or the mask-making process? 
Table 2: Domains With Student Statements

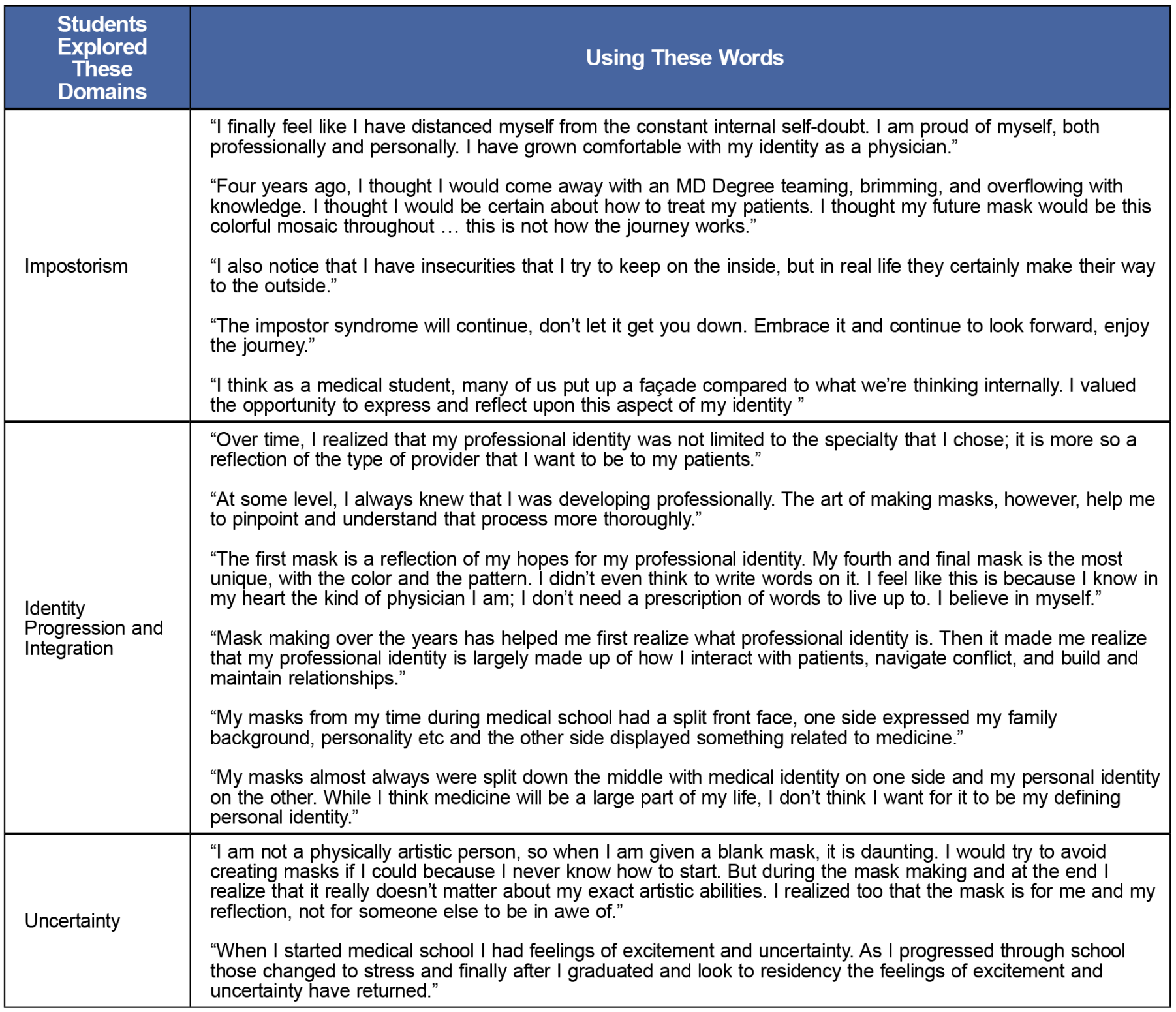


Figure 1: Representative Mask

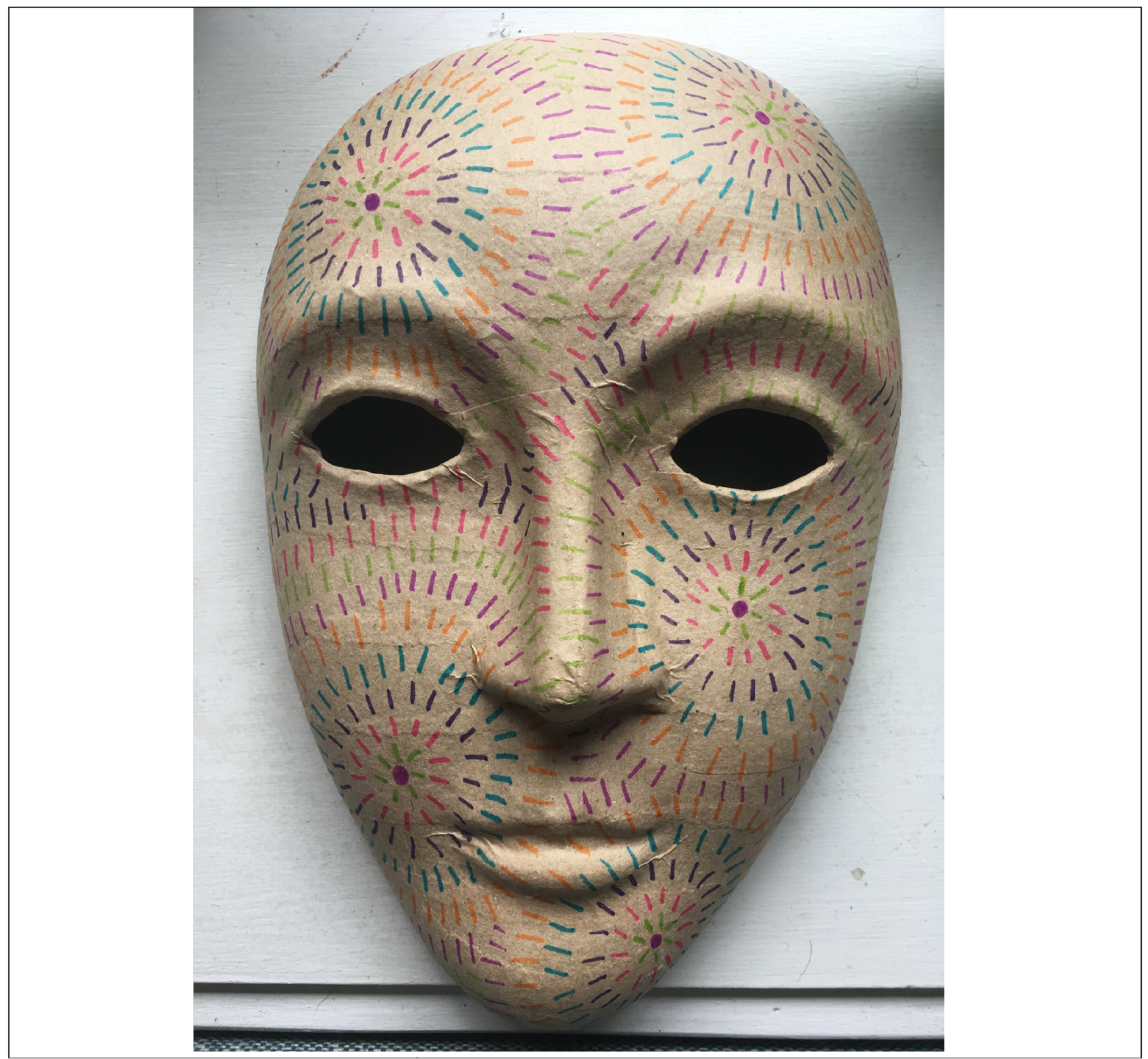

\section{Acknowledgments}

The author gratefully acknowledges the class of 2021 at the University Park Regional Campus of the Penn State College of Medicine.

\section{Corresponding Author}

Mark B. Stephens, MD, MS

1850 E. Park Ave, Suite 304, State College, PA 16803. 910-238-6101

Mstephens3@pennstatehealth.psu.edu

\section{Author Affiliations}

Mark B. Stephens, MD, MS - Department of Family and Community Medicine, and Department of Humanities, Penn State College of Medicine 


\section{References}

1. Dalia Y, Milam EC, Rieder EA. Art in medical education. J Grad Med Educ. 2020;12(6):686-695. doi:10.4300/JGME-D-20-00093.1

2. Bentwich ME, Gilbey P. More than visual literacy: art and the enhancement of tolerance for ambiguity and empathy. BMC Med Educ. 2017;17(1):200. doi:10.1186/s12909-017-1028-7

3. Joseph K, Bader K, Wilson S, Walker M, Stephens M, Varpio L. Unmasking identity dissonance: exploring medical students' professional identity formation through mask making. Perspect Med Educ. 2017;6(2):99-107. doi:10.1007/s40037-017-0339-z

4. Shapiro J, Youm J, Heare M, et al. Medical students' efforts to integrate and/or reclaim authentic identity: insights from a mask-making exercise. J Med Humanit. 2018;39(4):483-501. doi:10.1007/s10912-018-9534-0

5. Stephens MB, Bader KS, Myers KR, Walker MS, Varpio L. Examining professional identity formation through the ancient art of mask-making. J Gen Intern Med. 2019;34(7):1113-1115. doi:10.1007/s11606-019-04954-3

6. Stephens MB, Bowen JL, McGinley EL, Rainey P. Organizing chaos: iterative professional identity formation through the lens of mask making. PRiMER Peer-Rev Rep Med Educ Res. 2020;4:10. doi:10.22454/PRiMER.2020.705788

7. Kegan R. In Over Our Heads: The Mental Demands of Modern Life. Cambridge MA: Harvard University Press; 1994.

8. Cruess RL, Cruess SR, Boudreau JD, Snell L, Steinert Y. A schematic representation of the professional identity formation and socialization of medical students and residents: a guide for medical educators. Acad Med. 2015;90(6):718-725. doi:10.1097/ACM.0000000000000700

9. Goldie J. The formation of professional identity in medical students: considerations for educators. Med Teach. 2012;34(9):e641-e648. doi:10.3109/0142159X.2012.687476

10. Gallagher SR. Professional identity and imposter syndrome. Clin Teach. 2019;16(4):426-427. doi:10.1111/tct.13042

11. Gottlieb M, Chung A, Battaglioli N, Sebok-Syer SS, Kalantari A. Impostor syndrome among physicians and physicians in training: A scoping review. Med Educ. 2020;54(2):116-124. doi:10.1111/medu.13956

12. Bravata DM, Watts SA, Keefer AL, et al. Prevalence, predictors, and treatment of impostor syndrome: $A$ systematic review. J Gen Intern Med. 2020;35(4):1252-1275. doi:10.1007/s11606-019-05364-1

13. Nowell LS, Norris JM, White DE, Moules NJ. Thematic Analysis: striving to meet the trustworthiness criteria. Int J Qual Methods. 2017;16(1):1-13. doi:10.1177/1609406917733847

14. Houseknecht VE, Roman B, Stolfi A, Borges NJ. Longitudinal assessment of professional identity, wellness, imposter phenomenon and calling to medicine among medical students. Med Sci Educ. 2019;29(2):493-497. doi:10.1007/s40670-019-00718-0

15. Hatem DS, Halpin T. Becoming doctors: examining student narratives to understand the process of professional identity formation within a learning community. J Med Educ Curric Dev. 2019;6:2382120519834546. doi:10.1177/2382120519834546

Copyright $@ 2022$ by the Society of Teachers of Family Medicine 